\title{
Integrated Core-SOL-Divertor Modelling for ITER Including Impurity: Effect of Tungsten on Fusion Performance in H-mode and Hybrid Scenario
}

\author{
R. Zagórski ${ }^{1}$, I.Voitsekhovitch ${ }^{2}$, I. Ivanova-Stanik ${ }^{1}$, F. Köchl ${ }^{3}$, P.Belo ${ }^{2}$, J. Citrin ${ }^{4,5}$, E. Fable ${ }^{6}$, J. \\ Garcia $^{5}$, L. Garzotti ${ }^{2}$, J. Hobirk ${ }^{6}$, G. M. D. Hogeweij ${ }^{4}$, E. Joffrin ${ }^{5}$, X. Litaudon ${ }^{5}$, A. Polevoi ${ }^{7}$, \\ G. Telesca ${ }^{8}$ and JET contributors ${ }^{*}$ \\ JET-EFDA, Culham Science Centre, Abingdon, OX14 3DB, UK \\ ${ }^{1}$ Institute of Plasma Physics and laser Microfusion, Hery 23, 01-497 Warsaw, Poland \\ ${ }^{2}$ CCFE, Culham Science Centre, Abingdon, Oxon, OX14 3DB, UK \\ ${ }^{3}$ ÖAW/ATI, Atominstitut, TU Wien, 1020 Vienna, Austria \\ ${ }^{4}$ FOM Institute DIFFER - Dutch Institute for Fundamental Energy Research, The Netherlands \\ ${ }^{5}$ CEA, IRFM, F-13108 Saint-Paul-lez-Durance, France \\ ${ }^{6}$ Max-Planck-Institut für Plasmaphysik, D-85748 Garching, Germany \\ ${ }^{7}$ ITER Organization, Route de Vinon sur Verdon, 13115 St Paul Lez Durance, France \\ ${ }^{8}$ Department of Applied Physics, Ghent University, B-9000 Ghent, Belgium \\ * See the Appendix of F. Romanelli et al., Proceedings of the 24th IAEA Fusion Energy Conference 2012, San \\ Diego, USA \\ E-mail contact of main author: roman.zagorski@ipplm.pl
}

\begin{abstract}
The compatibility of two operational constraints - operation above the L-H power threshold and at low power to divertor - is examined for ITER long pulse H-mode and hybrid scenarios in integrated core-SOLdivertor modelling including impurities (intrinsic $\mathrm{Be}, \mathrm{He}, \mathrm{W}$ and seeded $\mathrm{Ne}$ ). The core thermal, particle and momentum transport is simulated with the GLF23 transport model tested in the self-consistent simulations of temperatures, density and toroidal rotation velocity in JET hybrid discharges and extrapolated to ITER. The beneficial effect of toroidal rotation velocity on fusion gain is shown. The sensitivity studies with respect to operational (separatrix and pedestal density, $\mathrm{Ne}$ gas puff) and unknown physics (W convective velocity and perpendicular diffusion in SOL as well as W prompt re-deposition) parameters are performed to determine their influence on the operational window and fusion gain.
\end{abstract}

\section{Introduction}

Different plasma performance (energy confinement, discharge duration) has been generally observed in JET discharges with carbon (C) and ITER-like wall (ILW) [1]. The presence of tungsten (W) in ILW discharges is one of the major changes introduced with the wall replacement which may partly explain the differences observed at JET and have an impact on ITER operation. The objective of this work is the estimation of ITER H-mode and hybrid performance during the burn phase in presence of $\mathrm{W}$ and other impurities [2] and identification of the operational domain, where high fusion gain $Q$ can be achieved. An impact of two important constraints - operation above the L-H power threshold and with low power to divertor - on the operational domain with high fusion gain is analysed here taking into account strong core-SOL-divertor coupling caused by W impurity. 


\section{Validation of physics models on existing experiments}

Validation of physics models against present experiments is a necessary step prior to their extrapolation to ITER. The GLF23 model for thermal and particle transport used here was validated on H-mode plasmas and hybrid scenarios (HS) performed on different tokamaks. The validation of this model in JET C wall HS with different toroidal rotation velocity $V_{\text {tor }}$, where the effect of the $E \times B$ shear on the confinement improvement can be clarified, was done recently in the self-consistent simulations including density, temperature and $V_{\text {tor }}$ (assuming $\chi_{\varphi}=\operatorname{Pr} \chi_{i}$, where $\operatorname{Pr}$ is the Prandtl number, $\chi_{\varphi}$ and $\chi_{i}$ are momentum and thermal diffusivity correspondingly) [3]. Addressing in particular to the $E \times B$ shear stabilisation of anomalous transport described by the linear turbulence quench rule in GLF23 model $\left(\gamma=\gamma_{\max }-\alpha_{E} \gamma_{E \times B}\right)$ the correlation of the amplification factor $\alpha_{E}$ with the toroidal rotation or Mach number was found (Fig. 1). The best agreement between the measured and simulated temperatures, density and toroidal rotation velocity in low density hybrid scenarios $\left(<n_{e}>=(2.5 \div 3.4) 10^{19} \mathrm{~m}^{-3}\right)$ was achieved by assuming $P r=0.3 \pm 0.05$ while larger Prandtl number with bigger uncertainties was found in the medium density hybrid discharge (\#77922) where a similarly large toroidal rotation velocity does not produce equally strong $E \times B$ shear stabilisation as in the low density discharges. These simulations determined the range of the GLF23 settings $\left(\alpha_{E}=0 \div 0.9\right.$ and $\operatorname{Pr}=0.3 \div 0.95)$ used in the modelling of ITER hybrid scenario. It should be mentioned that the $E \times B$ shear stabilisation is not a unique mechanism improving confinement in hybrid scenarios. Fast ion pressure gradient stabilisation [4], increase of the ITG threshold at high $s / q[5]$, nonlinear $\beta \mathrm{e}$ stabilisation [6], stabilisation of neoclassical tearing modes [7] also play an important role in certain experimental conditions. The COREDIV model (i.e. impurity transport in the core and SOL region, particle transport in $\mathrm{SOL}$, recycling coefficients for various species, impurity source) was successfully benchmarked with the JET H-mode discharges and advanced scenarios [8].

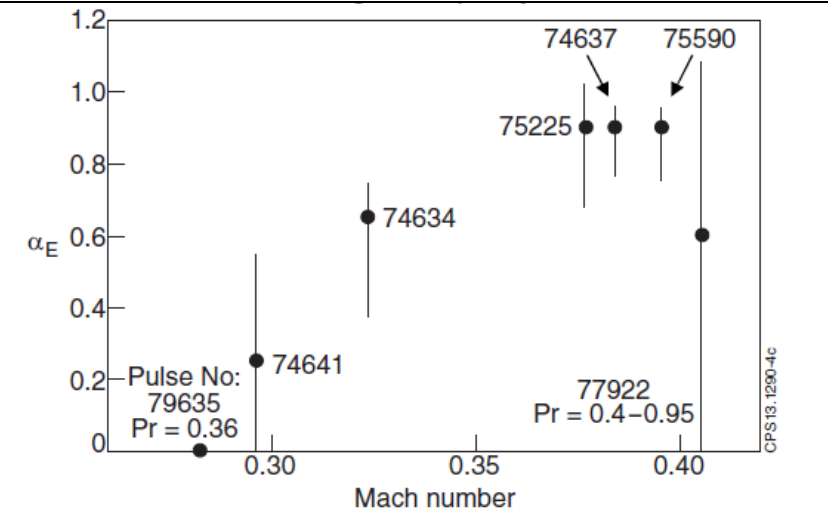

Fig 1. $\alpha_{E}$ values providing the most accurate prediction of $T_{e}, T_{i}, n_{i}$ and $V_{\text {tor }}$ in selected JET discharges vs. Mach number. Vertical lines show the $\alpha_{E}$ uncertainty limited by $15 \%$ deviation between simulated and measured quantities. Pr $=0.3$ is used for all discharges except \#79635 and \#77922 [3].

\section{Core-SOL-divertor model}

The effect of $\mathrm{W}$ on fusion performance is studied via integrated core-SOL-divertor modelling of main plasma species and impurities. ASTRA, JETTO and COREDIV codes are used here iteratively which allows one to combine the advanced transport, equilibrium, heating and current drive modules available in ASTRA and JETTO (Fokker-Planck NBI module, neoclassical current diffusion, theory-based heat and particle transport models) with the complex atomic physics and impurity (intrinsic $\mathrm{Be}, \mathrm{He}, \mathrm{W}$ and seeded $\mathrm{Ne}$ including their all charge states simulated self-consistently) simulations in the core, SOL and divertor regions (COREDIV). The impurity source (W sputtering at the divertor plate) is simulated here selfconsistently with the heat and particle fluxes from the plasma core to divertor (COREDIV) 
which determine the temperature and density at the divertor plate and therefore, the $\mathrm{W}$ sputtering yield. The sputtered W penetrates to the plasma core increasing the radiation and affecting the plasma performance (and therefore, the fluxes to the divertor). This non-linear coupling between core and divertor in presence of strongly radiating impurity may affect (i) the maintenance of the $\mathrm{H}$-mode

TABLE 1. Parameters of ITER long pulse H-mode and hybrid scenario used in present simulations

\begin{tabular}{|l|l|l|l|l|}
\hline & $\begin{array}{l}\mathrm{I}_{\mathrm{pl}} \\
{[\mathrm{MA}]}\end{array}$ & $\begin{array}{l}<\mathrm{n}_{\mathrm{e}}>, \mathrm{n}_{\text {ped }} \\
{\left[\mathrm{x} 10^{19} \mathrm{~m}^{-3}\right]}\end{array}$ & $\begin{array}{l}\mathrm{P}_{\mathrm{NBI}}, \mathrm{P}_{\mathrm{RF}} \\
{[\mathrm{MW}]}\end{array}$ & $\begin{array}{l}\mathrm{T}_{\mathrm{i}, \text { ped, }} \mathrm{T}_{\mathrm{e}, \mathrm{ped}}, \\
{[\mathrm{keV}]}\end{array}$ \\
\hline $\begin{array}{l}\text { Medium density } \\
\text { H-mode }\end{array}$ & 15 & $7.45,6.12$ & 33,20 & $7.5,6.7$ \\
\hline $\begin{array}{l}\text { High density } \\
\text { H-mode }\end{array}$ & 15 & $10.8,9$ & 33,20 & $4.8,4.4$ \\
\hline $\begin{array}{l}\text { Hybrid scenario } \\
\text { (zero tor. rotation) }\end{array}$ & 12 & $7.85,5.5$ & 33,37 & 5,5 \\
\hline $\begin{array}{l}\text { Hybrid scenario } \\
\text { (non-zero tor. } \\
\text { rotation, } P r=0.3)\end{array}$ & 12 & $8.3,5.5$ & 33,37 & 5,5 \\
\hline
\end{tabular}
pedestal when the power through the separatrix $P_{\text {loss }}$ is below the H-L transition power (which is assumed to be equal to L-H power threshold $P_{L H}$ and (ii) core performance and fusion gain. Another important constraint - low power to divertor plate with heat loads below $10 \mathrm{MW} / \mathrm{m}^{2}$ - must be taken into account in the estimations of operational domain. With simplified description of divertor geometry (COREDIV), the later constraint is roughly followed in simulations assuming that the total power to divertor plates $P_{\text {plate }}$ does not exceed $40 \mathrm{MW}$. With such assumption the present study will be concentrated mainly on the qualitative illustration of the effects of strong core - divertor coupling and investigation of the impact of various parameters on fusion performance via sensitivity studies.

\section{Effect of $W$ on fusion performance and power to divertor plate}

The parameters of ITER long-pulse H-mode [9] and HS used in coupled ASTRA/JETTOCOREDIV simulations are given in Table 1 . While the more detailed description of the results obtained for the H-mode scenarios can be found in ref. [10], the hybrid scenario is analysed in more detail here. The effect of the $E \times B$ shear on core confinement in hybrid scenario is illustrated in Fig. 2 by comparing the simulations performed with and without toroidal rotation velocity. A relatively low alpha heating $\left(P_{\alpha}=57.5 \mathrm{MW}\right)$ and fusion gain $(Q=4.2)$ have been obtained with the GLF23 model in the absence of toroidal rotation, while $Q$ increases to 5.8 in simulations with the most optimistic assumptions $\left(\alpha_{E}=0.9\right.$ and $\operatorname{Pr}=0.3$ ) validated on JET discharges 75225, 74637 and 75590 (Fig. 1). The impurity simulations performed for all three scenarios show similar $\mathrm{W}$ distribution, with low $\mathrm{W}$ charge states located near the edge and more stripped $\mathrm{W}$ dominating in the plasma core (Fig. 3 left). Correspondingly, two comparable peaks of $\mathrm{W}$ radiation produced by low (at the edge) and high (in the centre) $\mathrm{W}$ charge states have been obtained (Fig. 3 right).

Under assumptions used in COREDIV (similar impurity and main species transport) higher impurity density was obtained in HS with non-zero toroidal rotation velocity $\left(P r=0.3\right.$ and $\alpha_{E}$ $=0.9$ ) simulated with ASTRA as compared to the case with $V_{\text {tor }}=0$ (the density of main species is also higher in case with simulated $V_{\text {tor }}$, Fig. 2). As a consequence the radiative power $P_{\text {rad }}$ increases by $22 \%$, but such increase weakly affects the plasma performance.

Sensitivity of the core plasma performance to radiative power was further investigated by artificially re-scaling $P_{\text {rad }}$ by factor up to 5 in ASTRA simulations. Weak effect of radiation has been found in the power range limited by the total heating power (i.e. $P_{\text {rad }}<P_{a u x}+P_{\alpha}$ ) due to strong GLF23 stiffness (pedestal density and temperature were fixed in these simulations). 


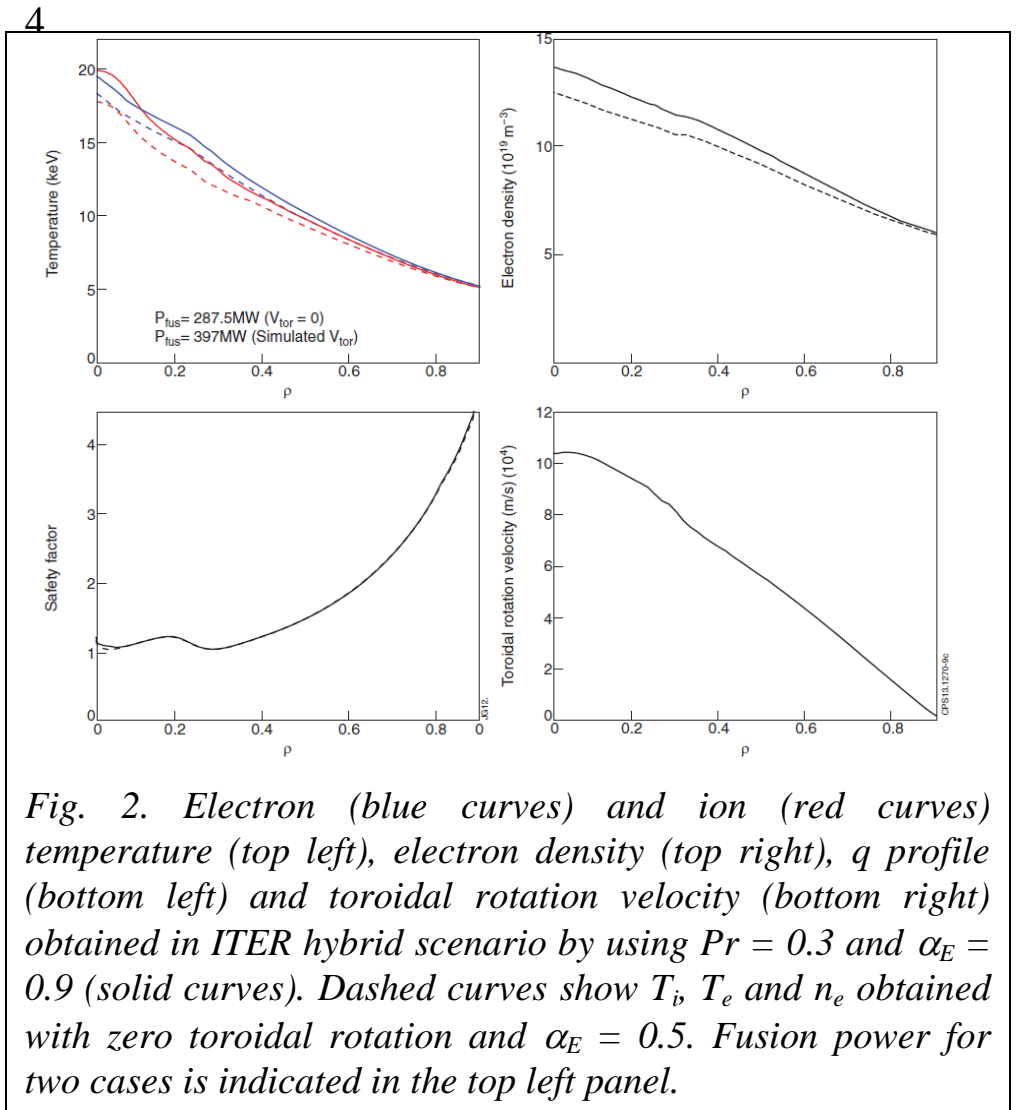

TH/P3-45

The operational constraints mentioned above (i.e., $P_{\text {loss }}>P_{L H}$ and $\left.P_{\text {plate }}<40 \mathrm{MW}\right)$ are compared for simulated H-mode plasmas and hybrid scenario in Table 2. The operation well above the L-H power threshold was achieved in all cases, but the power to divertor plate is very high. The possibility to reduce the power to divertor plate in these scenarios by applying the $\mathrm{Ne}$ seeding is analysed in next section

\section{Neon seeding}

Since the evolution of plasma parameters obtained with $\mathrm{Ne}$ seeding is qualitatively similar in
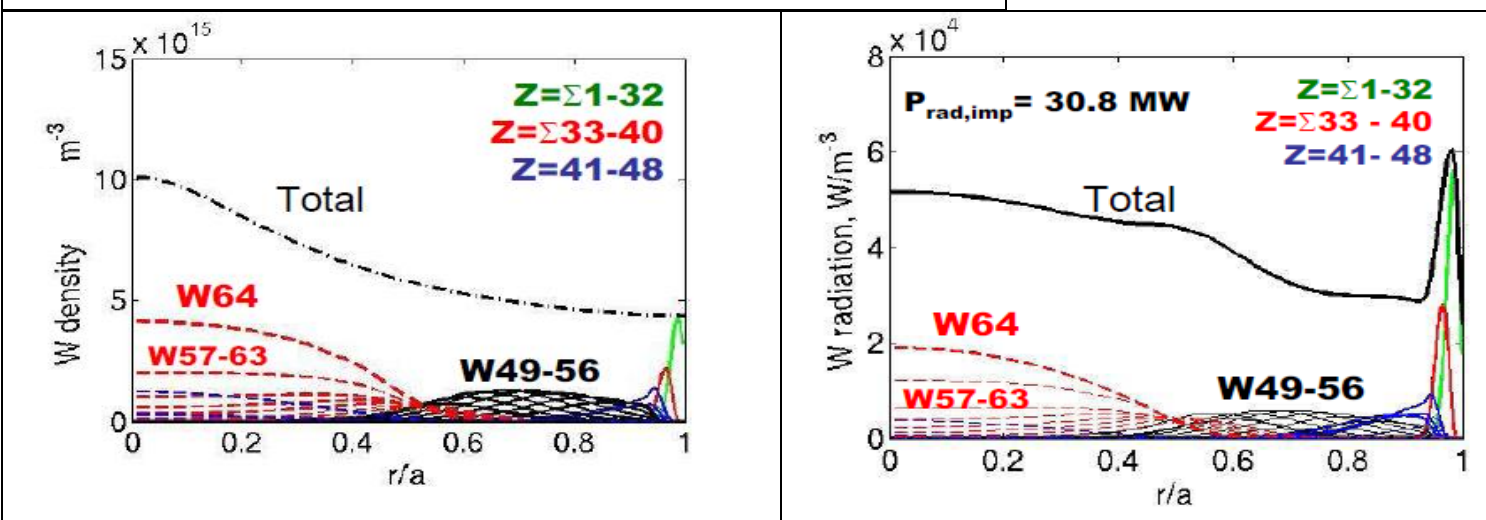

Fig. 3. Distribution of $W$ with different charge states (left) and $W$ radiation from different charge states (right) obtained in hybrid scenario.

all simulated scenarios we will concentrates here on one of them (HS) to illustrate the effect of seeding on the power to divertor plate and plasma performance. By adding the neon puff $\left(\Gamma_{N e}\right)$ and gradually increasing its concentration (same transport coefficients for $\mathrm{Ne}$ and main plasma species were used) the radiation in the edge and SOL regions was substantially increased (Fig. 4a), leading to a reduction of power to divertor plate (Fig. 4b). The temperature at the divertor plate strongly reduces with Ne puff (Fig. 4e) resulting in reduced $\mathrm{W}$ self-sputtering and sputtering by $\mathrm{D}$ and Be. However, the temperature at the plates was still large enough to cause high $\mathrm{W}$ sputtering by Ne. Consequently, the core $\mathrm{W}$ concentration and radiation increase at low $\Gamma_{N e}<1 \times 10^{21} \mathrm{~s}^{-1}$ (Fig. 4a,c). - Although the impact of W radiation on 
the core plasma performance is weak with stiff core transport, the power through the separatrix

reduces slightly below the L-H power threshold with low Ne puff and stays marginally above the L-H power threshold at $\Gamma_{N e}>$
TABLE 2. Power through the sepatarix $\left(\mathrm{P}_{\text {loss }}\right)$, L-H threshold power $\left(\mathrm{P}_{\mathrm{LH}}\right)$, total and core radiation and $\mathrm{Ne}$ influx providing the operation above the $\mathrm{L}-\mathrm{H}$ power threshold with power to divertor plate below $40 \mathrm{MW}\left(\mathrm{D}_{\mathrm{RAD}}{ }^{\mathrm{SOL}}=0.25 \mathrm{~m}^{2} / \mathrm{s}, \mathrm{V}_{\mathrm{imp}}=\mathrm{V}_{\mathrm{D}}\right.$ and $\mathrm{n}_{\text {sep }}=3.5 \times 10^{19} \mathrm{~m}^{-3}$ for $\mathrm{H}$-mode, $\mathrm{D}_{\mathrm{RAD}}{ }^{\mathrm{SOL}}=0.5 \mathrm{~m}^{2} / \mathrm{s}, \mathrm{V}_{\mathrm{imp}}=0$ and $\mathrm{n}_{\text {sep }}=3 \times 10^{19} \mathrm{~m}^{-3}$ for HS). Parameters achieved at the threshold Ne influx are shown in brackets.

\begin{tabular}{|l|l|l|l|l|l|l|}
\hline & $\begin{array}{l}\mathrm{P}_{\text {loss }} \\
{[\mathrm{MW}]}\end{array}$ & $\begin{array}{l}\mathrm{P}_{\mathrm{LH}} \\
{[\mathrm{MW}} \\
]\end{array}$ & $\begin{array}{l}\mathrm{P}_{\text {plate }} \\
{[\mathrm{MW}]}\end{array}$ & $\begin{array}{l}\mathrm{P}_{\mathrm{RAD}} \\
{[\mathrm{MW}]}\end{array}$ & $\begin{array}{l}\mathrm{P}_{\mathrm{RAD}} \text { CORE } \\
{[\mathrm{MW}]}\end{array}$ & $\begin{array}{l}\Gamma_{\mathrm{Ne}} \\
{\left[10^{21} \mathrm{~s}^{-1}\right]}\end{array}$ \\
\hline $\begin{array}{l}\text { Medium density } \\
\text { H-mode }\end{array}$ & $\begin{array}{l}91 \\
(62)\end{array}$ & 59.5 & $\begin{array}{l}76 \\
(37.4)\end{array}$ & $\begin{array}{l}58 \\
(84)\end{array}$ & $\begin{array}{l}42 \\
(69)\end{array}$ & $\begin{array}{l}0 \\
(1.25)\end{array}$ \\
\hline $\begin{array}{l}\text { High density H- } \\
\text { mode }\end{array}$ & 93.8 & 76.8 & 75.6 & 106 & 97.6 & 0 \\
\hline $\begin{array}{l}\text { Hybrid (zero } \\
\text { toroidal rotation) }\end{array}$ & $\begin{array}{l}96 \\
(70.7)\end{array}$ & 69 & $\begin{array}{l}70.8 \\
(32.7)\end{array}$ & $\begin{array}{l}55 \\
(95.4)\end{array}$ & $\begin{array}{l}44 \\
(75.5)\end{array}$ & $\begin{array}{l}0 \\
(2.1)\end{array}$ \\
\hline Hybrid (Pr=0.3) & $\begin{array}{l}112.7 \\
(72)\end{array}$ & 71.5 & $\begin{array}{l}92.6 \\
(39.7)\end{array}$ & $\begin{array}{l}62.4 \\
(114.6)\end{array}$ & $\begin{array}{l}51.4 \\
(94.3)\end{array}$ & $\begin{array}{l}0 \\
(1.5)\end{array}$ \\
\hline
\end{tabular}
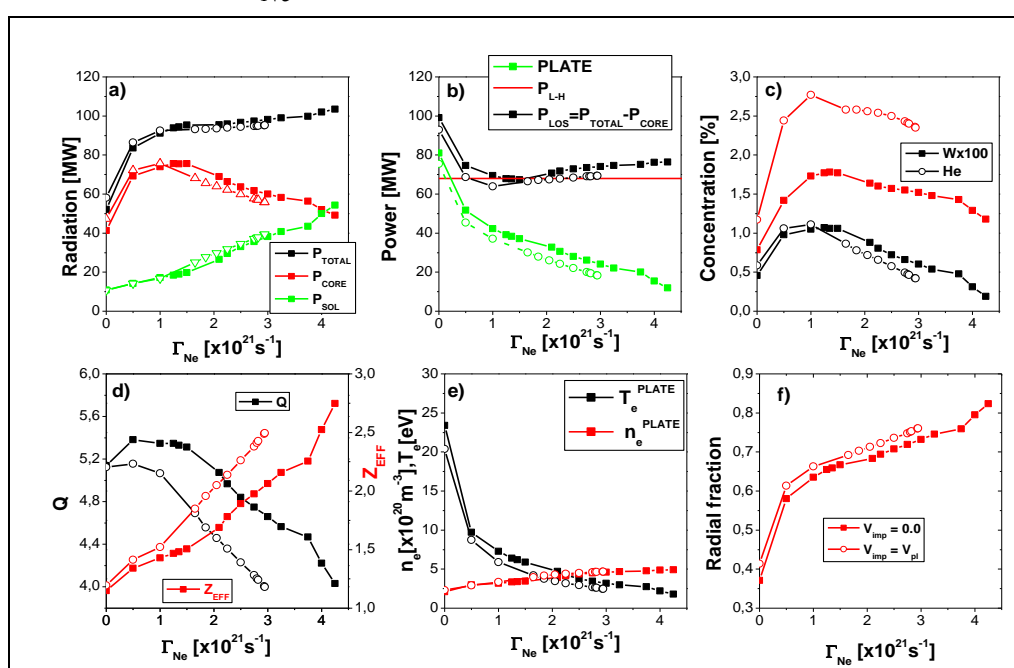

Fig. 4. Plasma parameters in ITER HS with zero rotation versus neon gas puff: (a) total, core and SOL radiative power; b) power to plate, $L-H$ power threshold and power through the separatrix; (c) $W$ and He concentrations (d), $Z_{E F F}$ and fusion gain $Q ;(d)$ temperature and density at the divertor plate and (e) radiation fraction. Reference separatrix electron density $n^{\text {sep }}{ }_{e}=3.0 \times 10^{19} \mathrm{~m}^{-3}$ and $D_{R A D}{ }^{S O L}=0.5 \mathrm{~m}^{2} / \mathrm{s}$

are used for two cases: $V_{\text {imp }}=0$ and $V_{\text {imp }}=V_{p l}$. investigated with
to find the operation above the L-H power threshold with low power to divertor plate.

\section{Sensitivity to $\mathrm{W}$ transport in core and $\mathrm{SOL}$ and $\mathrm{W}$ production at the divertor}

The effects of $\mathrm{W}$ core and SOL transport, $\mathrm{W}$ source and separatrix density on the fusion performance and operational constraints is studied by varying the $\mathrm{Ne}$ influx for selected parameter range while keeping all other parameters unchanged. 


\subsection{Effect of impurity convective velocity}

The reference simulations discussed in previous sections were performed assuming that the W convective velocity $V_{i m p}$ is similar to the deuterium convective velocity $V_{D}$ which in its turn has been simulated with the GLF23 model. As a consequence, similar deuterium and impurity peaking was obtained. The Ne gas puff scan repeated with $V_{\text {imp }}=0$ (Fig. 4, closed symbols) shows similar core W concentration and core radiation as compared to the case with GLF23-like W pinch, while the $\mathrm{He}$ concentration substantially reduces (similar convective velocity was used for all impurities) (Fig. 4c). The reduction of $\mathrm{He}$ concentration affects the dilution contributing to the increase of fusion gain (Fig. 4d). Although the power through
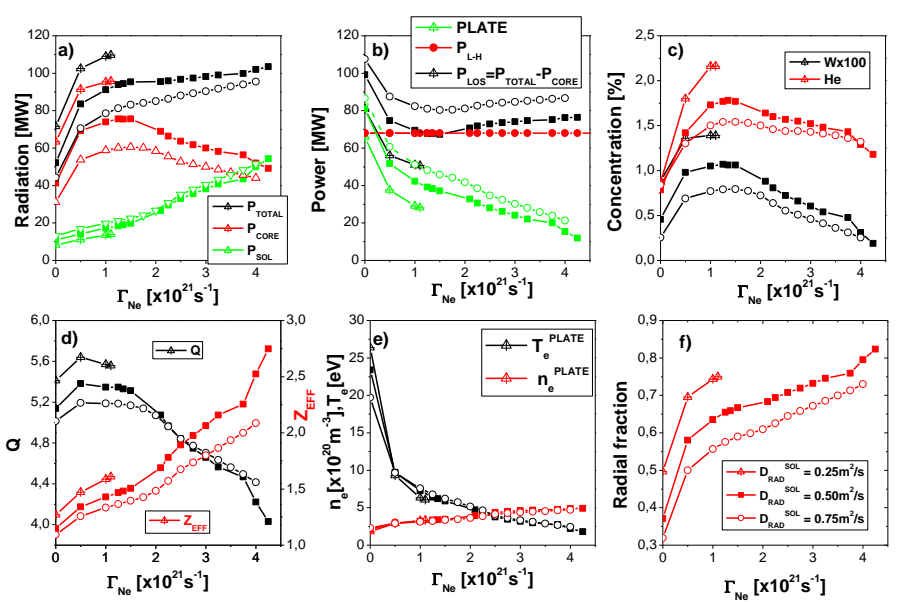

Fig. 5. Same plasma parameters as on Fig. 4 obtained with $n^{\text {sep }}{ }_{e}=$ $3.0 \times 10^{19} \mathrm{~m}^{-3}$ for three different values of the diffusion in SOL. the separatrix increases at zero $\mathrm{W}$ pinch the power to divertor plate increases as well. The operational constraints are satisfied at lower Ne gas puff $\left(\Gamma_{N e} \sim 2 \times 10^{21} 1 / \mathrm{s}\right)$ in simulations with $V_{\text {imp }}=0$ as compared to the reference simulations with $V_{i m p}=V_{D}$ illustrating the beneficial (but small) effect of zero impurity pinch. At high Ne gas puff the operation above the L-H power threshold, low temperature and high density at the divertor plate and low power to plate is achieved. It should be mentioned that outward W pinch at the edge and nearly zero pinch in the core has been obtained with NCLASS under given pedestal conditions.

\subsection{Effect of perpendicular diffusion coefficient in SOL}

Predictive COREDIV modelling of recent JET experiments with $\mathrm{N}$ and Ne seeding and high radiation in the SOL ( $>50 \%$ of total radiation) [11] shows that the better agreement with experimental data can be achieved by increasing the particle radial diffusion in SOL region $D_{R A D}{ }^{S O L}$ from $0.25 \mathrm{~m}^{2} / \mathrm{s}$ (this value was validated on standard $\mathrm{H}$-mode plasmas and used in the modelling of long pulse ITER H-mode [10]) to $0.5 \mathrm{~m}^{2} / \mathrm{s}$. The strong impact of $D_{R A D}{ }^{S O L}$ on plasma performance (improving the screening efficiency of SOL region) is illustrated on Fig. 5 . The core $\mathrm{W}$ concentration and radiation reduce with $D_{R A D}{ }^{S O L}$ leading to high power through separatrix $P_{\text {loss }}>P_{L H}$ with $D_{R A D} S O L>0.5 \mathrm{~m}^{2} / \mathrm{s}$ in the whole range of Ne gas puffs, but the operational window disappears completely at $D_{R A D}{ }^{S O L}=0.25 \mathrm{~m}^{2} / \mathrm{s}$. Fusion gain is weakly affected by SOL transport at high Ne gas puff where $P_{\text {plate }}<40 \mathrm{MW}$ and divertor conditions are close to detachment. The beneficial effect of $D_{R A D}{ }^{S O L}$ on operational window was also obtained in [12].

\subsection{Effect of separatrix density}

The variation of separatrix density in the range $(2.75-3.5) \times 10^{19} \mathrm{~m}^{-3}$ shows small beneficial effect of high density reducing the temperature at the divertor via thermal energy balance. The 
evolution of main plasma parameters with Ne puff is similar to one shown on Fig. 5 and not given here. The operational constraints $P_{\text {loss }}>P_{L H}$ and $P_{\text {plate }}<40 \mathrm{MW}$ are satisfied at $n_{\text {sep }}>$ $3 \times 10^{19} \mathrm{~m}^{-3}, D_{R A D} S O L>0.5 \mathrm{~m}^{2} / \mathrm{s}$ and $\Gamma_{N e}>2 \times 10^{21} 1 / \mathrm{s}$, with weak effect of separatrix density on the fusion gain in this range of $\mathrm{Ne}$ gas puff.

\subsection{Effect of prompt re-deposition}

Effect of promp re-deposition of sputtered $\mathrm{W}$ was neglected in previous simulations. This effect has been assessed by (i) artificial re-scaling of sputtered W outflux by factor $k_{\text {sput }}\left(k_{\text {sput }}\right.$ $=0.2 \div 1)$ and by introducing the expression for prompt redeposition [13] in COREDIV. The simulation results are presented in Fig. 6 . The $\mathrm{W}$ production reduces with decrease of the sputtering yield, reducing the radiation in the core and increasing the power through separatrix. The operation above the L-H power threshold is achieved in the whole range of $\mathrm{Ne}$ gas puff with $k_{\text {sput }}=0.5$ as well as with prompt re-deposition model. However, the power to plate increases with decreasing $k_{\text {sput }}$ emphasising

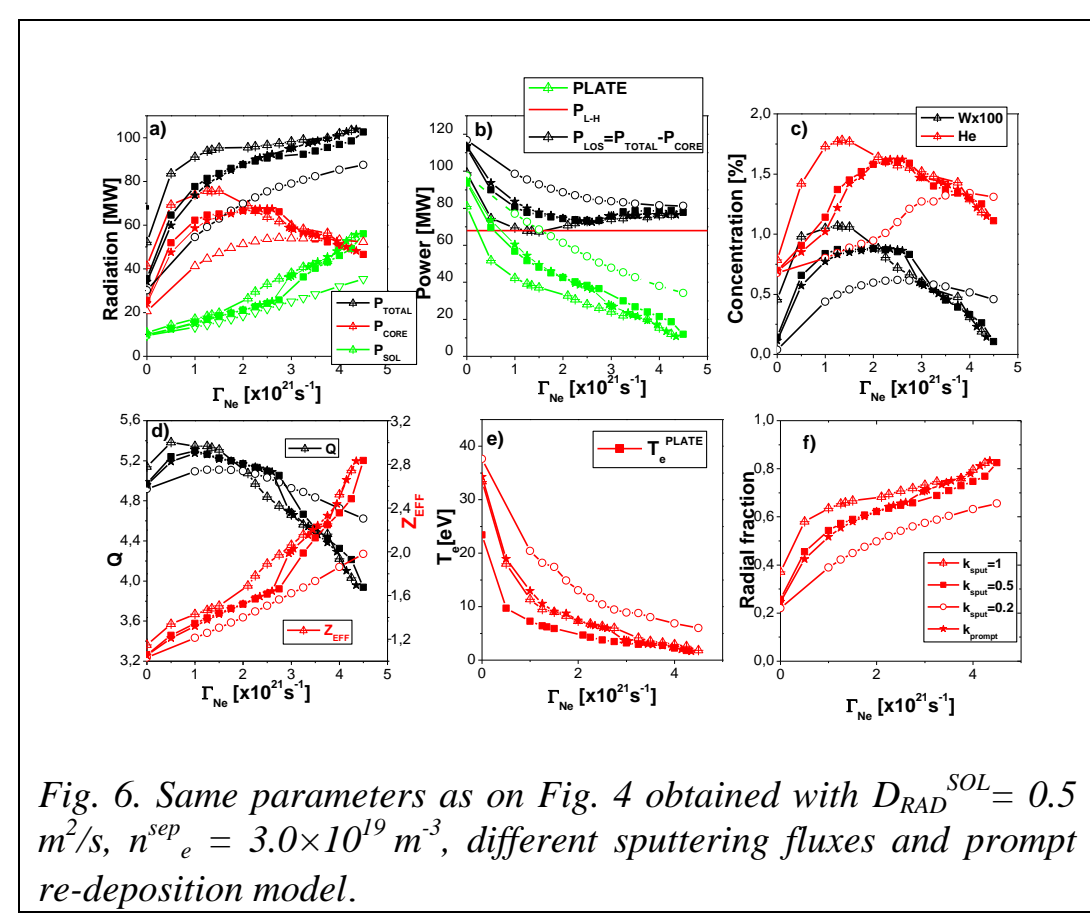
an importance of strong Ne puff. With standard sputtering model the acceptable level for $\mathrm{Ne}$ gas puff is $\Gamma_{N e}>1 \times 10^{21} \mathrm{~s}^{-1}$ which gives $Q=5.3$, but with the prompt re-deposition model the larger threshold in Ne gas puff $\Gamma_{N e}>2.25 \times 10^{21} \mathrm{~s}^{-1}$ is required resulting in smaller $Q=5.13$. The results achieved with prompt re-deposition model are similar to the reduction of the total sputtering yield by factor 2 . It should be noted, that larger reduction of the sputtering yield leads to the increase of divertor temperature and power to divertor plates that will require the operation at larger Ne gas puff (which at sufficiently high level might be incompatible with good fusion performance due to plasma dilution).

\section{Summary}

The compatibility of two operational constraints - operation above the L-H power threshold and at low power to divertor - is examined here in the integrated core-SOL-divertor modelling including impurities for ITER long pulse H-mode and hybrid scenarios. Such approach, which couples core and divertor plasmas, allows one to estimate self-consistently the effect of W accumulation and radiation on the power through the separatrix, core plasma performance, fluxes to divertor plate, divertor conditions and ultimately $\mathrm{W}$ production (the self-consistent treatment of core, SOL and divertor regions makes this approach different with [14]). The sensitivity studies with respect to operational (separatrix and pedestal density, Ne gas puff) and unknown physics (W transport and prompt re-deposition) parameters were performed to determine their influence on the operational window and fusion gain. With the assumptions 
used in present approach, the beneficial effects of reduced pedestal density (and therefore reduced $\left.P_{L H}\right)$ on the operational window $\left(P_{\text {loss }}>P_{L H}\right.$ and $P_{\text {plate }}<40 \mathrm{MW}$ ) was found even with low SOL particle diffusion $\left(D_{R A D} S O L=0.25 \mathrm{~m}^{2} / \mathrm{s}\right)$ [9]. The $\mathrm{W}$ pinch within the separatrix weakly affects the operational window, with a small positive (at zero or outward pinch) effect on $Q$. High $\mathrm{W}$ diffusion in SOL, high separatrix density and prompt $\mathrm{W}$ re-deposition largely extend the operational window, but require high Ne gas puff (particularly, when the prompt re-deposition, still known with large theoretical uncertainty, is sufficiently large) which leads to the reduction of fusion gain. In hybrid scenario the reduction of $Q$ with Ne gas puff is compensated by beneficial effect of the toroidal rotation velocity via the $E \times B$ shear stabilisation as well as other stabilising effects.

The results obtained here are based on few important assumptions such as fixed pedestal density and temperature and stiff transport model (GLF23). An impact of these assumptions on fusion performance and operational window needs to be further assessed in simulations with self-consistently computed pedestal transport and stability as well as less stiff transport models.

\section{Acknowledgement}

The authors would like to thank Dr R. Neu and Dr T. Pütterich from IPP Garching for providing them with atomic data for tungsten. This project has received funding from the European Union's Horizon 2020 research and innovation programme under grant agreement number 633053. This work was supported by EURATOM and carried out within the framework of the European Fusion Development Agreement. The views and opinions expressed herein do not necessarily reflect those of the European Commission.' This scientific work was financed within the Polish framework of the scientific financial resources in 2013 allocated for realization of the international co-financed project. This work has been partly done under ITER Scenario Modelling group of EFDA ITM Task Force.

[1] JOFFRIN E. et al, "Scenario development at JET with the new ITER-like wall" $24^{\text {th }}$ IAEA FEC Conf. 2012

[2] BREZINSEK S., "Plasma-surface interaction in the Be/W environment: conclusions drawn from the JETILW for ITER" $21^{\text {st }}$ PSI Conference.

[3] VOITSEKHOVITCH I. et al, "Modelling of JET hybrid scenarios with GLF23 transport model: E×B shear stabilization of anomalous transport" Nucl. Fusion 54 (2014) 093006

[4] GARCIA J. and GIRUZZI G., "On the different physical mechanisms for accessing hybrid scenarios on JET" Nucl. Fusion 53 (2013) 043023

[5] CITRIN J. et al "Predictive analysis of $q$-profile influence on transport in JET and ASDEX-Upgrade hybrid scenarios" Plasma Phys. Contr. Fusion 54 (2012) 065008

[6] CITRIN J. et al, "Nonlinear stabilization of tokamak microturbulence by fast ions" Phys. Rev. Lett. 111 (2012) 155001

[7] GUNTER S. et al "Neoclassical tearing modes on ASDEX Upgrade: improved scaling laws, high confinement at high $\beta_{\mathrm{N}}$ and new stabilization experiments" Nucl. Fusion 43 (2003) 161

[8] ZAGORSKI R. et al, "Integrated modelling of nitrogen seeded JET discharges" Contrib. Plasma Phys. 48 (13) (2008) 179

[9] POLEVOI A. et al, "Optimisation of ITER operational space for long-pulse scenarios" $40^{\text {th }}$ EPS Conf. on Plasma Phys., Espoo, Finland (2013), P2.135

[10] IVANOVA-STANIK I. et al, "Integrated core-SOL simulations of ITER H-mode scenarios with different pedestal density" Contrib. Plasma Phys. 54, No. 4-6, 341 - 346 (2014)

[11] WISCHMEIER, M. et.al "High density divertor operation for reactor relevant power exhaust", 21th Plasma Surfeace Interactions, Kanazawa, Japan, 26-30.05.2014, JAPAN

[12] KUKUSHKIN, A.,S.,et al., "Consequences of a reduction of the upstream power SOL width in ITER" J. Nuclear Materials, 438 (2013) S203

[13] DUX R, et al, "Main chamber sources and edge transport of tungsten in H-mode plasmas at ASDEX Upgrade" Nucl. Fusion, 51 (2011) 053002

[14] COSTER, D.P .et al., "SOLPS modelling of controlled ELMs for ITER",40th EPS Conference on Plasma Physics 